\title{
Role of shallow water waves generated by modified Camassa-Holm equation: A comparative analysis for traveling wave solutions
}

https://doi.org/10.1515/nleng-2021-0030

Received May 1, 2021; accepted Sep 16, 2021.

\begin{abstract}
There is no denying fact that harmonic crystals, cold plasma or liquids and compressible fluids are usually dependent of acoustic-gravity waves, acoustic waves, hydromagnetic waves, surface waves with long wavelength and few others. In this context, the exact solutions of the modified Camassa-Holm equation have been successfully constructed on the basis of comparative analysis of $\left(G^{\prime} / G-1 / G\right)$ and $\left(1 / G^{\prime}\right)$-expansion methods. The $\left(G^{\prime} / G-1 / G\right)$ and $\left(1 / G^{\prime}\right)$-expansion methods have been proved to be powerful and systematic tool for obtaining the analytical solutions of nonlinear partial differential equations so called modified Camassa-Holm equation. The solutions investigated via $\left(G^{\prime} / G-1 / G\right)$ and $\left(1 / G^{\prime}\right)$-expansion methods have remarkably generated trigonometric, hyperbolic, complex hyperbolic and rational traveling wave solutions. For the sake of different traveling wave solutions, we depicted 3-dimensional, 2dimensional and contour graphs subject to the specific values of the parameters involved in the governing equation. Two methods, which are important instruments in generating traveling wave solutions in mathematics, were compared both qualitatively and quantitatively. In addition, advantages and disadvantages of both methods are discussed and their advantages and disadvantages are revealed.
\end{abstract}

Keywords: $\left(G^{\prime} / G-1 / G\right)$-expansion method, $\left(1 / G^{\prime}\right)$ expansion method, modified Camassa-Holm equation, traveling wave solutions, comparative analysis

\footnotetext{
Asıf Yokuş, Department of Mathematics, Faculty of Science, Firat University, Elazig, 23200, Turkey

Hülya Durur, Department of Computer Engineering, Faculty of Engineering, Ardahan University, Ardahan, 75000, Turkey

*Corresponding Author: Kashif Ali Abro, Department of Ba-

sic Sciences and Related Studies, Mehran University of

Engineering and Technology, Jamshoro, Pakistan, E-mail:

kashif.abro@faculty.muet.edu.pk
}

\section{Introduction}

Nowadays, nonlinear partial differential equations have been applied in various fields due to their burning significances in fluid dynamics, optical fibers, biological sciences, quantum mechanics and plasma physics. Various researchers have suggested different methods to solve nonlinear partial differential equations such as, Homotopy perturbation method [1], Sine-Gordon expansion method [2, 3], (1/ $\left.G^{\prime}\right)$-expansion method [46], variational iteration method [7], improved Bernoulli sub-equation function method $[8,9],\left(1 / G^{\prime}\right)$-expansion method [10-12], (G'/G,1/G)-expansion method [13], Expfunction method [14], Auxiliary equation method [15], Laplace perturbation method [16], Adomian's decomposition method [17, 18], sub-equation method [19], Haar wavelet collocation method [20] and few others. In this context, Camassa-Holm equation is one of the type of nonlinear partial differential equation for which several studies have been presented in open literature; for instance; Gorka and Reyes [21] studied weak solutions and proved their existence and uniqueness for Camassa-Holm equation. Qu et al. [22] investigated dynamical stability of the single peaked soliton and periodic peaked soliton for an integrable Camassa-Holm equation with cubic nonlinearity. The soliton wave solutions using homotopy analysis method for Camassa-Holm equation have been explored by Abbasbandy [23]. Bekir and Guner [24] suggested new study based on topological (dark) soliton solutions subject to solitary wave Ansatz method for Camassa-Holm equation [24]. In brevity, few recent studies can be viewed in [25-27]. Additionally, the recent work on exact [28-32, 34, 35] and analytical solutions can also be seen therein [36-42]. Motivated by above discussion, we have been traced out analytic solutions of the Camassa-Holm equation by using $\left(G^{\prime} / G-1 / G\right)$ and $\left(1 / G^{\prime}\right)$-expansion methods. We also presented the comparison of $\left(G^{\prime} / G-1 / G\right)$ and $\left(1 / G^{\prime}\right)$-expansion methods on Camassa-Holm equation. The Camassa-Holm equation can be written in the form of [43]

$$
u_{t}-u_{x x t}+3 u^{2} u_{x}-2 u_{x} u_{x x}-u u_{x x x}=0 .
$$


Eq. (1) possesses shallow water waves and such mathematical model is known to be integrable, possessing multisoliton solutions with peaks [44-46]. Additionally, several fractional analytical techniques can be persuaded with classical and non-classical [47-58], local and nonlocal [59-67] and singular kernels [68-74]. In this study, general information about the equations and analytical methods discussed in the introduction is given. In the second section, the general operation of analytical methods is explained. In the third section, the applications of the methods are carried out. In the fourth section, the advantages and disadvantages of the obtained data and methods are discussed. In the last section, the data in the study is compiled.

\section{Comparative methods for Camassa-Holm equation}

\section{$2.1\left(1 / G^{\prime}\right)$-Expansion method}

We consider two-variable general form of nonlinear partial differential equations

$$
P\left(u, \frac{\partial u}{\partial t}, \frac{\partial u}{\partial x}, \frac{\partial^{2} u}{\partial x^{2}}, \ldots\right)=0
$$

in the general form. Here, let $u=u(x, t)=U(\xi), \quad \xi=$ $x+v t, \quad v \neq 0$, where $v$ is a constant and the velocity of the wave. After this, it can be converted into following nonlinear ordinary differential equation for $U(\xi)$ :

$$
y\left(U, U^{\prime}, U^{\prime \prime}, U^{\prime \prime \prime}, \ldots\right)=0
$$

The solution of Eq. (3) is assumed to have the form

$$
U(\xi)=a_{0}+\sum_{i=1}^{m} a_{i}\left(\frac{1}{G^{\prime}}\right)^{i},
$$

where $a_{i}, \quad(i=1,2,3, \ldots, m$.$) are constants, m$ is a positive integer, which is balancing term in Eq. (2), and $G=$ $G(\xi)$ provides the following second order ordinary differential equation as:

$$
G^{\prime \prime}+\lambda G^{\prime}+\mu=0
$$

where $\lambda$ and $\mu$ are constants to be determined after,

$$
\frac{1}{G^{\prime}(\xi)}=\frac{1}{-\frac{\mu}{\lambda}+A \cosh [\xi \lambda]-A \sinh [\xi \lambda]},
$$

where $A$ is constant. The Eq. (5a) is a solution of the Eq. (5). If the desired derivatives of the Eq. (4) are calculated and substituting in the Eq. (3), a polynomial with the argument $\left(1 / G^{\prime}\right)$ is attained. An algebraic equation system is created by equalizing the coefficients of this polynomial to zero. This equation system is solved with the help of ready package program and put into place in the default Eq. (3) for solution function. Consequently, the solutions of the Eq. (1) are found.

\section{$2.2\left(G^{\prime} / G-1 / G\right)$-Expansion method}

The form of nonlinear partial differential equation containing two or more independent variables for which the solution can be explored by using $\left(G^{\prime} / G-1 / G\right)$ expansion method is written as follows:

$$
K\left(u, u_{t}, u_{x}, u_{y}, u_{z}, u_{t t}, u_{x x}, \ldots\right)=0
$$

If $u=u(x, t)=U(\xi), \quad \xi=x+v t$ transformations are used in Eq. (6) then $v$ is a constant, Eq. (6) is converted into a nonlinear ordinary differential equation and this equation can be generally written as:

$$
f\left(U, U^{\prime}, U^{\prime \prime}, U^{\prime \prime \prime}, \ldots\right)=0 .
$$

Here, Eq. (7) can be integrated to decrease the operational complexity. By the nature of $\left(G^{\prime} / G-1 / G\right)$-expansion method, $G(\xi)$ function is solution function of the second order ordinary differential equation as

$$
G^{\prime \prime}(\xi)+\lambda G(\xi)=\mu,
$$

where $\lambda$ and $\mu$ are real constants. As, $\phi=\phi(\xi)=G^{\prime} / G$ and $\psi=\psi(\xi)=\frac{1}{G(\xi)}$ provides operational esthetic. We can write the derivatives of the functions defined herein as;

$$
\phi^{\prime}=-\phi^{2}+\mu \psi-\lambda, \psi^{\prime}=-\phi \psi
$$

We can present the behaviors of the solution functions of Eq. (8) with respect to the condition of $\lambda$ by considering the equations given by Eq. (9).

Case I: If $\lambda<0$

$$
G(\xi)=c_{1} \sinh (\sqrt{-\lambda} \xi)+c_{2} \cosh (\sqrt{-\lambda} \xi)+\frac{\mu}{\lambda},
$$

whereas $c_{1}$ and $c_{2}$ are arbitrary constants. By considering Eq. (10);

$$
\psi^{2}=\frac{-\lambda}{\lambda^{2} \sigma+\mu^{2}}\left(\phi^{2}-2 \mu \psi+\lambda\right), \quad \sigma=c_{1}^{2}-c_{2}^{2} .
$$

Eq. (11) is easily written.

Case II: If $\lambda>0$

$$
G(\xi)=c_{1} \sin (\sqrt{\lambda} \xi)+c_{2} \cos (\sqrt{\lambda} \xi)+\frac{\mu}{\lambda},
$$


here $c_{1}$ and $c_{2}$ are arbitrary constants. By considering Eq. (12), there is following equation;

$$
\psi^{2}=\frac{\lambda}{\lambda^{2} \sigma-\mu^{2}}\left(\phi^{2}-2 \mu \psi+\lambda\right), \quad \sigma=c_{1}^{2}+c_{2}^{2},
$$

Case III: If $\lambda=0$

$$
G(\xi)=\frac{\mu}{2} \xi^{2}+c_{1} \xi+c_{2},
$$

here $c_{1}$ and $c_{2}$ are arbitrary constants. By considering Eq. (14), there is following equation;

$$
\psi^{2}=\frac{1}{c_{1}^{2}-2 \mu c_{2}}\left(\phi^{2}-2 \mu \psi\right) .
$$

Finally, the solution of Eq. (7) in terms of $\phi$ and $\psi$ polynomials is expressed as;

$$
U(\xi)=\sum_{i=0}^{m} a_{i} \phi^{i}+\sum_{i=1}^{m} b_{i} \phi^{i-1} \psi
$$

Here, $a_{i}(i=0,1, \ldots, m)$ and $b_{i}(i=1, \ldots, m)$ numbers are the constants to be determined later. $m$ is a positive equilibrium term which can be attained by comparing maximum order derivative with the maximum order nonlinear term in Eq. (7). If Eq. (16) is written in Eq. (7) along with Eqs. $(9,11,13)$ or $(15)$, a polynomial function related to $\phi$ and $\psi$ is written. Each coefficient of $\phi^{i} \psi^{j}(i=0,1, \ldots, m)(j=1, \ldots, m)$ terms of the attained polynomial functions are equated to zero and an algebraic equation system is attained for $a_{i}, b_{i}, v, \mu, c_{1}, c_{2}$ and $\lambda(i=0,1, \ldots, m)$. The required coefficients are found by solving this algebraic equation by means of ready package program. These coefficients found are put into Eq. (16) and $U(\xi)$ solution function of the ordinary differential equation given as Eq. (7) is attained and if $\xi=x+v t$ transformation is operated in reverse order, we will obtain the desired $u(x, t)$ traveling wave solution of Eq. (6).

\section{Solutions of modified Camassa-Holm equation}

\section{$3.1\left(G^{\prime} / G-1 / G\right)$-Expansion method}

We consider Camassa-Holm Eq. (1). Using transmutation $u=u(x, t)=U(\xi), \quad \xi=x+v t$ and taking once the integral of Eq. (1), we get

$$
v\left(U-U^{\prime \prime}\right)+U^{3}-\frac{1}{2}\left(U^{\prime}\right)^{2}-U U^{\prime \prime}=0 .
$$

Where, vis the wave velocity. Thus, by finding the equilibrium term $m=2$ in Eq. (17), and in Eq. (16) we obtain to following form of the solution

$$
U(\xi)=a_{0}+a_{1} \phi[\xi]+b_{1} \psi[\xi]+a_{2} \phi[\xi]^{2}+b_{2} \phi[\xi] \psi[\xi] .
$$

If we substitute the Eq. (18) in the Eq. (17) and the coefficients of the algebraic equation are equal to zero, we can establish the following algebraic equation systems

$$
\begin{aligned}
\text { Const } & : v a_{0}+a_{0}^{3}-\frac{1}{2} \lambda^{2} a_{1}^{2}-\frac{\lambda^{2} \mu^{2} a_{1}^{2}}{2\left(-\mu^{2}+\lambda^{2} \sigma\right)}-2 v \lambda^{2} a_{2}-\frac{2 v \lambda^{2} \mu^{2} a_{2}}{-\mu^{2}+\lambda^{2} \sigma}-2 \lambda^{2} a_{0} a_{2} \\
& -\frac{2 \lambda^{2} \mu^{2} a_{0} a_{2}}{-\mu^{2}+\lambda^{2} \sigma}+\frac{v \lambda^{2} \mu b_{1}}{-\mu^{2}+\lambda^{2} \sigma}+\frac{\lambda^{2} \mu a_{0} b_{1}}{-\mu^{2}+\lambda^{2} \sigma}+\frac{4 \lambda^{3} \mu a_{2} b_{1}}{-\mu^{2}+\lambda^{2} \sigma}-\frac{\lambda^{3} b_{1}^{2}}{-\mu^{2}+\lambda^{2} \sigma}+\frac{3 \lambda^{2} a_{0} b_{1}^{2}}{-\mu^{2}+\lambda^{2} \sigma}+\frac{2 \lambda^{3} \mu a_{1} b_{2}}{-\mu^{2}+\lambda^{2} \sigma}-\frac{\lambda^{4} b_{2}^{2}}{2\left(-\mu^{2}+\lambda^{2} \sigma\right)}=0,
\end{aligned}
$$

$\phi[\xi]: \quad v a_{1}-2 v \lambda a_{1}-2 \lambda a_{0} a_{1}+3 a_{0}^{2} a_{1}-4 \lambda^{2} a_{1} a_{2}-\frac{4 \lambda^{2} \mu^{2} a_{1} a_{2}}{-\mu^{2}+\lambda^{2} \sigma}+\frac{5 \lambda^{2} \mu a_{1} b_{1}}{-\mu^{2}+\lambda^{2} \sigma}+\frac{3 \lambda^{2} a_{1} b_{1}^{2}}{-\mu^{2}+\lambda^{2} \sigma}+\frac{6 v \lambda^{2} \mu b_{2}}{-\mu^{2}+\lambda^{2} \sigma}+\frac{6 \lambda^{2} \mu a_{0} b_{2}}{-\mu^{2}+\lambda^{2} \sigma}$ $+\frac{8 \lambda^{3} \mu a_{2} b_{2}}{-\mu^{2}+\lambda^{2} \sigma}-\frac{7 \lambda^{3} b_{1} b_{2}}{-\mu^{2}+\lambda^{2} \sigma}+\frac{6 \lambda^{2} a_{0} b_{1} b_{2}}{-\mu^{2}+\lambda^{2} \sigma}=0$, 


$$
\begin{aligned}
& (\phi[\xi])^{2}: \quad-3 \lambda a_{1}^{2}-\frac{\lambda \mu^{2} a_{1}^{2}}{2\left(-\mu^{2}+\lambda^{2} \sigma\right)}+3 a_{0} a_{1}^{2}+v a_{2}-8 v \lambda a_{2}-\frac{2 v \lambda \mu^{2} a_{2}}{-\mu^{2}+\lambda^{2} \sigma}-8 \lambda a_{0} a_{2} \\
& -\frac{2 \lambda \mu^{2} a_{0} a_{2}}{-\mu^{2}+\lambda^{2} \sigma}+3 a_{0}^{2} a_{2}-4 \lambda^{2} a_{2}^{2}-\frac{4 \lambda^{2} \mu^{2} a_{2}^{2}}{-\mu^{2}+\lambda^{2} \sigma}+\frac{v \lambda \mu b_{1}}{-\mu^{2}+\lambda^{2} \sigma}+\frac{\lambda \mu a_{0} b_{1}}{-\mu^{2}+\lambda^{2} \sigma} \\
& +\frac{17 \lambda^{2} \mu a_{2} b_{1}}{-\mu^{2}+\lambda^{2} \sigma}-\frac{7 \lambda^{2} b_{1}^{2}}{2\left(-\mu^{2}+\lambda^{2} \sigma\right)}+\frac{3 \lambda a_{0} b_{1}^{2}}{-\mu^{2}+\lambda^{2} \sigma}+\frac{3 \lambda^{2} a_{2} b_{1}^{2}}{-\mu^{2}+\lambda^{2} \sigma}+\frac{14 \lambda^{2} \mu a_{1} b_{2}}{-\mu^{2}+\lambda^{2} \sigma} \\
& +\frac{6 \lambda^{2} a_{1} b_{1} b_{2}}{-\mu^{2}+\lambda^{2} \sigma}-\frac{15 \lambda^{3} b_{2}^{2}}{2\left(-\mu^{2}+\lambda^{2} \sigma\right)}+\frac{3 \lambda^{2} a_{0} b_{2}^{2}}{-\mu^{2}+\lambda^{2} \sigma}=0 \\
& (\phi[\xi])^{3}:: \quad-2 v a_{1}-2 a_{0} a_{1}+a_{1}^{3}-14 \lambda a_{1} a_{2}-\frac{4 \lambda \mu^{2} a_{1} a_{2}}{-\mu^{2}+\lambda^{2} \sigma}+6 a_{0} a_{1} a_{2}+\frac{5 \lambda \mu a_{1} b_{1}}{-\mu^{2}+\lambda^{2} \sigma} \\
& +\frac{3 \lambda a_{1} b_{1}^{2}}{-\mu^{2}+\lambda^{2} \sigma}+\frac{6 v \lambda \mu b_{2}}{-\mu^{2}+\lambda^{2} \sigma}+\frac{6 \lambda \mu a_{0} b_{2}}{-\mu^{2}+\lambda^{2} \sigma}+\frac{30 \lambda^{2} \mu a_{2} b_{2}}{-\mu^{2}+\lambda^{2} \sigma}-\frac{17 \lambda^{2} b_{1} b_{2}}{-\mu^{2}+\lambda^{2} \sigma} \\
& +\frac{6 \lambda a_{0} b_{1} b_{2}}{-\mu^{2}+\lambda^{2} \sigma}+\frac{6 \lambda^{2} a_{2} b_{1} b_{2}}{-\mu^{2}+\lambda^{2} \sigma}+\frac{3 \lambda^{2} a_{1} b_{2}^{2}}{-\mu^{2}+\lambda^{2} \sigma}=0 \\
& (\phi[\xi])^{4}:: \quad-\frac{5 a_{1}^{2}}{2}-6 v a_{2}-6 a_{0} a_{2}+3 a_{1}^{2} a_{2}-12 \lambda a_{2}^{2}-\frac{4 \lambda \mu^{2} a_{2}^{2}}{-\mu^{2}+\lambda^{2} \sigma}+3 a_{0} a_{2}^{2} \\
& +\frac{13 \lambda \mu a_{2} b_{1}}{-\mu^{2}+\lambda^{2} \sigma}-\frac{5 \lambda b_{1}^{2}}{2\left(-\mu^{2}+\lambda^{2} \sigma\right)}+\frac{3 \lambda a_{2} b_{1}^{2}}{-\mu^{2}+\lambda^{2} \sigma}+\frac{12 \lambda \mu a_{1} b_{2}}{-\mu^{2}+\lambda^{2} \sigma} \\
& +\frac{6 \lambda a_{1} b_{1} b_{2}}{-\mu^{2}+\lambda^{2} \sigma}-\frac{15 \lambda^{2} b_{2}^{2}}{-\mu^{2}+\lambda^{2} \sigma}+\frac{3 \lambda a_{0} b_{2}^{2}}{-\mu^{2}+\lambda^{2} \sigma}+\frac{3 \lambda^{2} a_{2} b_{2}^{2}}{-\mu^{2}+\lambda^{2} \sigma}=0 \\
& (\phi[\xi])^{5}:: \quad-10 a_{1} a_{2}+3 a_{1} a_{2}^{2}+\frac{22 \lambda \mu a_{2} b_{2}}{-\mu^{2}+\lambda^{2} \sigma}-\frac{10 \lambda b_{1} b_{2}}{-\mu^{2}+\lambda^{2} \sigma}+\frac{6 \lambda a_{2} b_{1} b_{2}}{-\mu^{2}+\lambda^{2} \sigma}+\frac{3 \lambda a_{1} b_{2}^{2}}{-\mu^{2}+\lambda^{2} \sigma}=0 \\
& (\phi[\xi])^{6}:: \quad-8 a_{2}^{2}+a_{2}^{3}-\frac{8 \lambda b_{2}^{2}}{-\mu^{2}+\lambda^{2} \sigma}+\frac{3 \lambda a_{2} b_{2}^{2}}{-\mu^{2}+\lambda^{2} \sigma}=0,
\end{aligned}
$$

$\psi[\xi]:: \quad \lambda \mu a_{1}^{2}+\frac{\lambda \mu^{3} a_{1}^{2}}{-\mu^{2}+\lambda^{2} \sigma}+4 v \lambda \mu a_{2}+\frac{4 v \lambda \mu^{3} a_{2}}{-\mu^{2}+\lambda^{2} \sigma}+4 \lambda \mu a_{0} a_{2}+\frac{4 \lambda \mu^{3} a_{0} a_{2}}{-\mu^{2}+\lambda^{2} \sigma}$

$$
\begin{aligned}
& +v b_{1}-v \lambda b_{1}-\frac{2 v \lambda \mu^{2} b_{1}}{-\mu^{2}+\lambda^{2} \sigma}-\lambda a_{0} b_{1}-\frac{2 \lambda \mu^{2} a_{0} b_{1}}{-\mu^{2}+\lambda^{2} \sigma}+3 a_{0}^{2} b_{1}-2 \lambda^{2} a_{2} b_{1}-\frac{8 \lambda^{2} \mu^{2} a_{2} b_{1}}{-\mu^{2}+\lambda^{2} \sigma} \\
& +\frac{2 \lambda^{2} \mu b_{1}^{2}}{-\mu^{2}+\lambda^{2} \sigma}-\frac{6 \lambda \mu a_{0} b_{1}^{2}}{-\mu^{2}+\lambda^{2} \sigma}-\lambda^{2} a_{1} b_{2}-\frac{4 \lambda^{2} \mu^{2} a_{1} b_{2}}{-\mu^{2}+\lambda^{2} \sigma}+\frac{\lambda^{3} \mu b_{2}^{2}}{-\mu^{2}+\lambda^{2} \sigma}=0,
\end{aligned}
$$

$\phi[\xi] \psi[\xi]: \quad 3 v \mu a_{1}+3 \mu a_{0} a_{1}+8 \lambda \mu a_{1} a_{2}+\frac{8 \lambda \mu^{3} a_{1} a_{2}}{-\mu^{2}+\lambda^{2} \sigma}-4 \lambda a_{1} b_{1}-\frac{10 \lambda \mu^{2} a_{1} b_{1}}{-\mu^{2}+\lambda^{2} \sigma}+6 a_{0} a_{1} b_{1}$

$$
\begin{aligned}
& -\frac{6 \lambda \mu a_{1} b_{1}^{2}}{-\mu^{2}+\lambda^{2} \sigma}+v b_{2}-5 v \lambda b_{2}-\frac{12 v \lambda \mu^{2} b_{2}}{-\mu^{2}+\lambda^{2} \sigma}-5 \lambda a_{0} b_{2}-\frac{12 \lambda \mu^{2} a_{0} b_{2}}{-\mu^{2}+\lambda^{2} \sigma} \\
& +3 a_{0}^{2} b_{2}-4 \lambda^{2} a_{2} b_{2}-\frac{16 \lambda^{2} \mu^{2} a_{2} b_{2}}{-\mu^{2}+\lambda^{2} \sigma}+\frac{14 \lambda^{2} \mu b_{1} b_{2}}{-\mu^{2}+\lambda^{2} \sigma}-\frac{12 \lambda \mu a_{0} b_{1} b_{2}}{-\mu^{2}+\lambda^{2} \sigma}=0,
\end{aligned}
$$

$\phi[\xi]^{2} \psi[\xi] \quad: 4 \mu a_{1}^{2}+10 v \mu a_{2}+10 \mu a_{0} a_{2}+8 \lambda \mu a_{2}^{2}+\frac{8 \lambda \mu^{3} a_{2}^{2}}{-\mu^{2}+\lambda^{2} \sigma}-2 v b_{1}-2 a_{0} b_{1}+3 a_{1}^{2} b_{1}$

$$
\begin{aligned}
& -11 \lambda a_{2} b_{1}-\frac{26 \lambda \mu^{2} a_{2} b_{1}}{-\mu^{2}+\lambda^{2} \sigma}+6 a_{0} a_{2} b_{1}+\frac{5 \lambda \mu b_{1}^{2}}{-\mu^{2}+\lambda^{2} \sigma}-\frac{6 \lambda \mu a_{2} b_{1}^{2}}{-\mu^{2}+\lambda^{2} \sigma}-10 \lambda a_{1} b_{2} \\
& -\frac{24 \lambda \mu^{2} a_{1} b_{2}}{-\mu^{2}+\lambda^{2} \sigma}+6 a_{0} a_{1} b_{2}-\frac{12 \lambda \mu a_{1} b_{1} b_{2}}{-\mu^{2}+\lambda^{2} \sigma}+\frac{14 \lambda^{2} \mu b_{2}^{2}}{-\mu^{2}+\lambda^{2} \sigma}-\frac{6 \lambda \mu a_{0} b_{2}^{2}}{-\mu^{2}+\lambda^{2} \sigma}=0,
\end{aligned}
$$

$\phi[\xi]^{3} \psi[\xi] \quad:: \quad 17 \mu a_{1} a_{2}-5 a_{1} b_{1}+6 a_{1} a_{2} b_{1}-6 v b_{2}-6 a_{0} b_{2}+3 a_{1}^{2} b_{2}-19 \lambda a_{2} b_{2}$

$$
-\frac{44 \lambda \mu^{2} a_{2} b_{2}}{-\mu^{2}+\lambda^{2} \sigma}+6 a_{0} a_{2} b_{2}+\frac{20 \lambda \mu b_{1} b_{2}}{-\mu^{2}+\lambda^{2} \sigma}-\frac{12 \lambda \mu a_{2} b_{1} b_{2}}{-\mu^{2}+\lambda^{2} \sigma}-\frac{6 \lambda \mu a_{1} b_{2}^{2}}{-\mu^{2}+\lambda^{2} \sigma}=0,
$$

$\phi[\xi]^{4} \psi[\xi] \quad:: \quad 14 \mu a_{2}^{2}-10 a_{2} b_{1}+3 a_{2}^{2} b_{1}-10 a_{1} b_{2}+6 a_{1} a_{2} b_{2}+\frac{16 \lambda \mu b_{2}^{2}}{-\mu^{2}+\lambda^{2} \sigma}-\frac{6 \lambda \mu a_{2} b_{2}^{2}}{-\mu^{2}+\lambda^{2} \sigma}=0$,

$$
\phi[\xi]^{5} \psi[\xi] \quad:: \quad-16 a_{2} b_{2}+3 a_{2}^{2} b_{2}=0,
$$




$$
\begin{gathered}
\psi[\xi]^{3}:: \quad-2 \mu^{2} a_{2} b_{1}+\mu b_{1}^{2}+b_{1}^{3}-\mu^{2} a_{1} b_{2}+\lambda \mu b_{2}^{2}=0, \\
\phi[\xi] \psi[\xi]^{3}: \quad-4 \mu^{2} a_{2} b_{2}+8 \mu b_{1} b_{2}+3 b_{1}^{2} b_{2}=0, \\
\phi[\xi]^{2} \psi[\xi]^{3}: \quad 8 \mu b_{2}^{2}+3 b_{1} b_{2}^{2}=0, \\
\phi[\xi]^{3} \psi[\xi]^{3}: \quad b_{2}^{3}=0, \\
\psi[\xi]^{3} \quad: \quad-2 \mu^{2} a_{2} b_{1}+\mu b_{1}^{2}+b_{1}^{3}-\mu^{2} a_{1} b_{2}+\lambda \mu b_{2}^{2}=0,
\end{gathered}
$$

aims with ready package program, reaching the solutions of system (19) then we obtained the following cases:

Case I: If $\lambda>0$,

$a_{0}=1, a_{1}=0, a_{2}=8, b_{1}=0, b_{2}=0, v=-1, \mu=0, \lambda=\frac{1}{4}$,

replacing the values of Eq. (20) into Eq. (18) then we have the following trigonometric traveling wave solution for Eq. (1)

$$
\xi=x+v t
$$

$u_{1}(x, t)=1+\frac{8\left(\frac{1}{2} c_{2} \cos \left[\frac{1}{2}(-t+x)\right]-\frac{1}{2} c_{1} \sin \left[\frac{1}{2}(-t+x)\right]\right)^{2}}{\left(c_{1} \cos \left[\frac{1}{2}(-t+x)\right]+c_{2} \sin \left[\frac{1}{2}(-t+x)\right]\right)^{2}}$

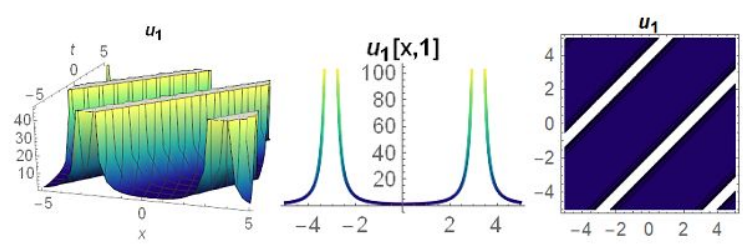

Figure 1: 3-D, 2-D and contour graphs for $c_{2}=0.5, c_{1}=-1$ values in Eq. (21).

Case II: If $\lambda<0$,

$a_{0}=-2, a_{1}=0, a_{2}=8, b_{1}=0, b_{2}=0, v=-2, \mu=0, \lambda=-\frac{1}{4}$,

replacing Eq. (22) into Eq. (18) then we obtain the following hyperbolic traveling wave solution of Eq. (1):

$$
\xi=x+v t,
$$

$$
\begin{aligned}
& u_{2}(x, t)=-2+ \\
& \frac{8\left(\frac{1}{2} c_{2} \cosh \left[\frac{1}{2}(-2 t+x)\right]+\frac{1}{2} c_{1} \sinh \left[\frac{1}{2}(-2 t+x)\right]\right)^{2}}{\left(c_{1} \cosh \left[\frac{1}{2}(-2 t+x)\right]+c_{2} \sinh \left[\frac{1}{2}(-2 t+x)\right]\right)^{2}} .
\end{aligned}
$$

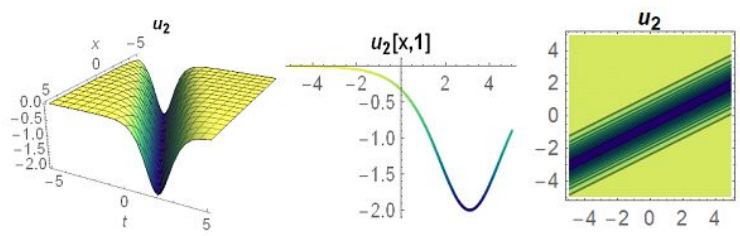

Figure 2: 3-D, 2-D and contour graphs for $c_{2}=0.5, c_{1}=-1$ values in Eq. (23).

Case III: If $\lambda=0$,

$$
a_{0}=1, a_{1}=0, a_{2}=8, b_{1}=0, b_{2}=0, v=-1, \mu=0 \text {, }
$$

replacing values of Eq. (24) into Eq. (18), then we obtain the following rational traveling wave solution for Eq. (1):

$$
\begin{gathered}
\xi=x+v t, \\
u_{3}(x, t)=\frac{8 c_{2}{ }^{2}}{\left(c_{1}+c_{2} x\right)^{2}} .
\end{gathered}
$$
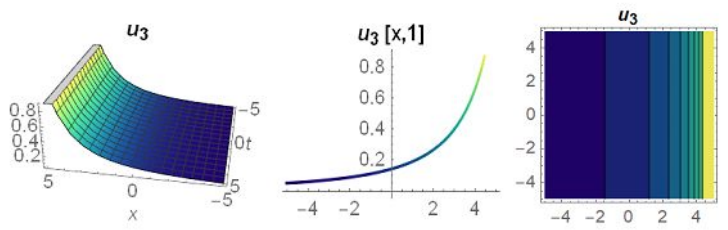

Figure 3: 3-D, 2-D and contour graphs for $c_{2}=-0.2, \quad c_{1}=1.5$ values in Eq. (25).

\section{$3.2\left(1 / G^{\prime}\right)$-Expansion method}

We consider Eq. (1). For which using transmutation $u=$ $u(x, t)=U(\xi), \quad \xi=x+v t, \quad v \neq 0$, and taking once the integral of Eq. (1), we obtain

$$
v\left(U-U^{\prime \prime}\right)+U^{3}-\frac{1}{2}\left(U^{\prime}\right)^{2}-U U^{\prime \prime}=0,
$$

where, $v$ represents the velocity of the wave. Taking into account the Eq. (26), we find the equilibrium term $m=2$ and in Eq. (4), we attain to following form of the solution

$$
U(\xi)=a_{0}+a_{1}\left(\frac{1}{G^{\prime}}\right)+a_{2}\left(\frac{1}{G^{\prime}}\right)^{2} .
$$


If we substitute the Eq. (27) in the Eq. (26) and the coefficients of the algebraic equation are equal to zero, we can establish the following algebraic equation systems

$$
\begin{gathered}
\text { Const : } v a_{0}+a_{0}^{3}=0, \\
\left(\frac{1}{G^{\prime}[\xi]}\right)^{1}: v a_{1}-v \lambda^{2} a_{1}-\lambda^{2} a_{0} a_{1}+3 a_{0}^{2} a_{1}=0, \\
\left(\frac{1}{G^{\prime}[\xi]}\right)^{2}: \quad-3 v \lambda \mu a_{1}-3 \lambda \mu a_{0} a_{1}-\frac{3}{2} \lambda^{2} a_{1}^{2}+3 a_{0} a_{1}^{2}+v a_{2}-4 v \lambda^{2} a_{2}-4 \lambda^{2} a_{0} a_{2}+3 a_{0}^{2} a_{2}=0, \\
\left(\frac{1}{G^{\prime}[\xi]}\right)^{3}: \quad-2 v \mu^{2} a_{1}-2 \mu^{2} a_{0} a_{1}-4 \lambda \mu a_{1}^{2}+a_{1}^{3}-10 v \lambda \mu a_{2}-10 \lambda \mu a_{0} a_{2}-7 \lambda^{2} a_{1} a_{2}+6 a_{0} a_{1} a_{2}=0, \\
\left(\frac{1}{G^{\prime}[\xi]}\right)^{4}:-\frac{5}{2} \mu^{2} a_{1}^{2}-6 v \mu^{2} a_{2}-6 \mu^{2} a_{0} a_{2}-17 \lambda \mu a_{1} a_{2}+3 a_{1}^{2} a_{2}-6 \lambda^{2} a_{2}^{2}+3 a_{0} a_{2}^{2}=0, \\
\left(\frac{1}{G^{\prime}[\xi]}\right)^{5}:-10 \mu^{2} a_{1} a_{2}-14 \lambda \mu a_{2}^{2}+3 a_{1} a_{2}^{2}=0, \\
\left(\frac{1}{G^{\prime}[\xi]}\right)^{6}:-8 \mu^{2} a_{2}^{2}+a_{2}^{3}=0 .
\end{gathered}
$$

Case I: If

$$
a_{0}=-1, \quad a_{1}= \pm 8 \text { i } \mu, \quad a_{2}=8 \mu^{2}, \quad v= \pm 1, \quad \lambda=-\mathrm{i}, \quad(\mathrm{i}=\sqrt{-1}),
$$

replacing values Eq. (29) into Eq. (27) and we have the following new type complex hyperbolic traveling wave solution for Eq. (1):

$$
\begin{gathered}
\xi=x+v t, \\
u_{4}(x, t)=-1+\frac{8 \mu^{2}}{(-\mathrm{i} \mu+A \cos [t-x]-\mathrm{iA} \sin [t-x])^{2}}-\frac{8 \mathrm{i} \mu}{-\mathrm{i} \mu+A \cos [t-x]-\mathrm{iA} \sin [t-x]} .
\end{gathered}
$$

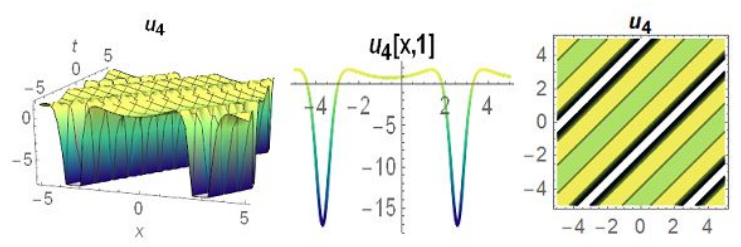

Figure 4: 3-D, 2-D and contour graphs for $A=0.5, \mu=1$ values in Eq. (30).

Case II: If

$$
a_{0}=0, \quad a_{1}=-8 \mu, \quad a_{2}=8 \mu^{2}, \quad v=-2, \quad \lambda=-1,
$$

replacing values of Eq. (31) into Eq. (27) and we have following hyperbolic traveling wave solution for Eq. (1):

$$
\begin{gathered}
\xi=x+v t, \\
u_{2}(x, t)=\frac{8 \mu^{2}}{(\mu+A \cosh [2 t-x]-A \sinh [2 t-x])^{2}}-\frac{8 \mu}{\mu+A \cosh [2 t-x]-A \sinh [2 t-x]} .
\end{gathered}
$$




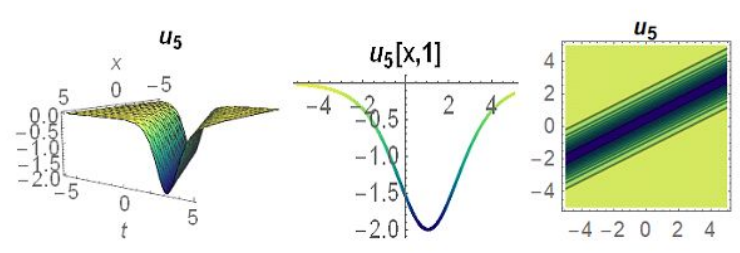

Figure 5: 3-D, 2-D and contour graphs for $A=5, \mu=1.9$ values in Eq. (32).

\section{Results and discussions}

Shock waves of nonlinear partial differential equations (NLPDEs) have been discussed on the basis of modeling of physical phenomena. The comparative analysis has been attained by two methods namely $\left(G^{\prime} / G-1 / G\right)$ expansion and $\left(1 / G^{\prime}\right)$-expansion method for CamassaHolm equation. It has been traced out that the solutions are different than the existing solutions in literature. This assured that the results have disclosed new phenomenon for Shock waves based on two different methods. For the sake of physical aspects, it provides the opportunity to understand the dynamics of solitary waves obtained by two expansion methods their states. The solutions obtained with the $\left(G^{\prime} / G-1 / G\right)$-expansion method are trigonometric, hyperbolic, and rational traveling wave solutions. From comparison point of view, only hyperbolic and complex hyperbolic traveling wave solutions have been obtained via $\left(1 / G^{\prime}\right)$-expansion method. The solutions obtained by both methods were found to be different from each other. In this case, the existence of many methods expresses the richness of the solutions of the differential equation. $\left(G^{\prime} / G-1 / G\right)$-expansion method more complicated and $\left(1 / G^{\prime}\right)$-expansion method is less difficult. In this case, we can determine the degree of difficulty by referring to the system of Eq. (19) and (28). It was also observed that the processing time in $\left(G^{\prime} / G-1 / G\right)$ expansion method was longer by using a ready package program with the same features. The excess of the number of equations in the equation system (19) is effective on the extension of period. It has been observed that all obtained exact solutions, the $\left(1 / G^{\prime}\right)$-expansion method is advantages in terms of process complexity, while $\left(G^{\prime} / G-1 / G\right)$ expansion method is more advantages in terms of number of solutions.

In this study, the application of two different analytical methods is included, and the solutions obtained at the end of this application are important both mathematically and physically. Mathematically important is the generation of traveling wave solutions. Physically, traveling wave solutions, which play an important role in the transport of energy, will shed light on many problems. If the parameters in the traveling wave solution gain physical meaning by considering the physical properties of the problem under consideration, the obtained traveling wave solutions will be much more valuable. It was observed that the traveling wave solutions obtained by both analytical methods satisfy the modified Camassa-Holm equation. At the end of this observation, it can be said that the methods are reliable, useful and applicable methods for obtaining traveling wave solution. Both methods are recommended for obtaining traveling wave solution of NLPDEs in the future.

\section{Conclusion}

In this letter, as a result, trigonometric, hyperbolic, complex hyperbolic and rational traveling wave solutions of modified Camassa-Holm equation have successfully constructed using $\left(G^{\prime} / G-1 / G\right)$ and $\left(1 / G^{\prime}\right)$ expansion methods. 3-D, 2-D and contour graphs are presented for the arbitrary values of the parameters in the solutions obtained. The solutions obtained by both methods have different properties and can shed light on some physical events such as different shallow water waves. Advantages and disadvantages of two methods discussed. In the future, it can be used to find traveling wave solutions of many NLPDEs. Because both methods are powerful methods for obtaining traveling wave solutions of NLPDEs.

Acknowledgements: Author Asıf Yokuş is very grateful to Firat University, Elazig, Turkey, Hülya Durur is very grateful Ardahan University, Ardahan, Turkey and Kashif Ali Abro are highly thankful and grateful to Mehran university of Engineering and Technology, Jamshoro, Pakistan for generous support and facilities of this research work.

Conflict of interest: The authors declare no conflict of interest.

Data Availability Statement: The data that support the findings of this study are available from the corresponding author upon reasonable request. 


\section{References}

[1] He JH. Homotopy perturbation method: a new nonlinear analytical technique. Appl Math Comput. 2003;135(1)3-9.

[2] Baskonus HM, Bulut H, Sulaiman TA. New Complex Hyperbolic Structures to the Lonngren-Wave Equation by Using Sine-Gordon Expansion Method. Appl Math Nonlinear Sci. 2019;4(1):129-38.

[3] Yokus A, Sulaiman TA, Bulut H. On the analytical and numerical solutions of the Benjamin-Bona-Mahony equation. Opt Quantum Electron. 2018;50(1):31.

[4] Yokus A, Durur H, Ahmad H. Hyperbolic type solutions for the couple Boiti-Leon-Pempinelli system. Facta Univ Ser Math Inform. 2020;35:523-31.

[5] Durur H, Yokuş A. Exact solutions of the Benney-Luke equation via (1/G/)-expansion method. Bilecik Şeyh Edebali Üniversitesi Fen Bilimleri Dergisi. 2021;8(1):56-64.

[6] Yokus A, Durur H, Ahmad H, Yao SW. Construction of Different Types Analytic Solutions for the Zhiber-Shabat Equation. Mathematics. 2020;8(6):908.

[7] He JH. Variational iteration method for autonomous ordinary differential systems. Appl Math Comput. 2000;114(2-3):11523.

[8] Baskonus HM, Bulut H. On the complex structures of KunduEckhaus equation via improved Bernoulli sub-equation function method. Waves Random Complex Media. 2015;25(4):7208.

[9] Dusunceli F, Celik E, Askin M. New exact solutions for the doubly dispersive equation using an improved Bernoulli subequation function method. Indian J Phys. 2018;95(2):309-314.

[10] Naher H, Abdullah FA, Akbar MA. The (G//G)-Expansion Method for Abundant Traveling Wave Solutions of CaudreyDodd-Gibbon Equation. Math Probl Eng. 2011.

[11] Wang M, Li X, Zhang J. The (G/G)-expansion method and travelling wave solutions of nonlinear evolution equations in mathematical physics. Phys Lett A. 2008;372(4):417-23.

[12] Durur H. Different types analytic solutions of the (1+1)dimensional resonant nonlinear Schrödinger's equation using (G//G)-expansion method. Mod Phys Lett B. 2020;34(3):2050036.

[13] Yokuş A, Durur H, Abro KA, Kaya D. Role of Gilson-Pickering equation for the different types of soliton solutions: a nonlinear analysis. Eur Phys J Plus. 2020;135(8):1-19.

[14] Esen A, Kutluay S. Application of the Exp-function method to the twodimensional sine-Gordon equation. Int J Nonlinear Sci Numer Simul. 2009;10(10):1355-60.

[15] Jiong S. Auxiliary equation method for solving nonlinear partial differential equations. Phys Lett A. 2003;309(5-6):38796.

[16] . Yavuz M, Ozdemir N, Baskonus HM. Solutions of partial differential equations using the fractional operator involving Mittag-Leffler kernel. Eur Phys J Plus. 2018;133(6):215.

[17] Kaya D, Yokus A. A numerical comparison of partial solutions in the decomposition method for linear and nonlinear partial differential equations. Math Comput Simul. 2002;60(6):50712.

[18] Kaya D, Yokus A. A decomposition method for finding solitary and periodic solutions for a coupled higher-dimensional Burgers equations. Appl Math Comput. 2005;164(3):857-64.
[19] Duran S, Karabulut B. Nematicons in liquid crystals with Kerr Law by sub-equation method. Alex Eng J. 2021. https://doi.org/10.1016/j.aej.2021.06.077.

[20] Aziz I, Amin R. Numerical solution of a class of delay differential and delay partial differential equations via Haar wavelet. Appl Math Model. 2016;40(23-24):10286-99.

[21] Górka P, Reyes EG. The modified Camassa-Holm equation. Int Math Res Not. 2011;2011(12):2617-49.

[22] Qu C, Liu X, Liu Y. Stability of peakons for an integrable modified Camassa-Holm equation with cubic nonlinearity. Commun Math Phys. 2013;322(3):967-97.

[23] Abbasbandy S. Solitary wave solutions to the modified form of Camassa-Holm equation by means of the homotopy analysis method. Chaos Solitons Fractals. 2009;39(1):428-35.

[24] Bekir A, Güner Ö. Topological (dark) soliton solutions for the Camassa-Holm type equations. Ocean Eng. 2013;74:276-9.

[25] He B. New peakon, solitary wave and periodic wave solutions for the modified Camassa-Holm equation. Nonlinear Analysis: theory. Methods \& Applications. 2009;71(12):6011-8.

[26] Gao A, Shen C. Optimal solution for the viscous modified Camassa-Holm equation. Journal of Nonlinear Mathematical Physics. 2010;17(4):571-89.

[27] Gao Y, Liu JG. Global Convergence of a Sticky Particle Method for the Modified Camassa-holm Equation. SIAM J Math Anal. 2017;49(2):1267-94.

[28] Durur H, Yokuş A. Discussions on diffraction and the dispersion for traveling wave solutions of the $(2+1)$-dimensional paraxial wave equation. Math Sci. 2021;1-11.

[29] Al-Mdallal QM, Syam MI. Sine-Cosine method for finding the soliton solutions of the generalized fifth-order nonlinear equation. Chaos Solitons Fractals. 2007;33(5):1610-7.

[30] Hamid M, Usman M, Zubair T, Haq RU, Shafee A. An efficient analysis for $\mathrm{N}$-soliton, Lump and lump-kink solutions of timefractional (2+1)-Kadomtsev-Petviashvili equation. Physica A. 2019;528:121320.

[31] Barman HK, Aktar MS, Uddin MH, Akbar MA, Baleanu D, Osman MS. Physically significant wave solutions to the Riemann wave equations and the Landau-Ginsburg-Higgs equation. Results Phys. 2021;27:104517.

[32] Duran S, Yokuş A, Durur H, Kaya D. Refraction simulation of internal solitary waves for the fractional Benjamin-Ono equation in fluid dynamics. Mod Phys Lett B. 2021;35(26):2150363.

[33] Durur H. Energy-carrying wave simulation of the Lonngrenwave equation in semiconductor materials. Int J Mod Phys B. 2021;35(21):2150213.

[34] Duran S. Travelling wave solutions and simulation of the Lonngren wave equation for tunnel diode. Opt Quantum Electron. 2021;53(8):1-9.

[35] Duran S. Breaking theory of solitary waves for the Riemann wave equation in fluid dynamics. Int J Mod Phys B. 2021;35(9):2150130.

[36] Özkan YS, Seadawy AR, Yaşar E. On the optical solitons and local conservation laws of Chen-Lee-Liu dynamical wave equation. Optik (Stuttg). 2021;227:165392.

[37] Abro KA. Role of fractal-fractional derivative on ferromagnetic fluid via fractal Laplace transform: A first problem via fractal-fractional differential operator. Eur J Mech BFluids. 2021;85:76-81.

[38] Bhojraj L, Kashif AA, Abdul WS. Thermodynamical analysis of heat transfer of gravity-driven fluid flow via frac- 
tional treatment: an analytical study. J Therm Anal Calorim. 2020; https://doi.org/10.1007/s10973-020-09429-w.

[39] Abro KA, Abdon A. A comparative study of convective fluid motion in rotating cavity via Atangana-Baleanu and CaputoFabrizio fractal-fractional differentiations. Eur Phys J Plus. 2020;135(2):226-42.

[40] Ali AK, Ambreen S, Abdon A. Thermal stratification of rotational second-grade fluid through fractional differential operators. J Therm Anal Calorim. 2020; https://doi.org/10.1007/s10973-020-09312-8.

[41] Kashif AA, Abdon A. Role of Non-integer and Integer Order Differentiations on the Relaxation Phenomena of Viscoelastic Fluid. Phys Scr. 2020;95(3):035228.

[42] Abro K A, A Fractional and Analytic Investigation of ThermoDiffusion Process on Free Convection Flow: An Application to Surface Modification Technology, European Phys J Plus. 2020;135(1):31-45 https://doi.org/10.1140/epjp/s13360-01900046-7.

[43] Aslan I, Öziş T. Analytic study on two nonlinear evolution equations by using the $(\mathrm{G} / \mathrm{G})$-expansion method. Appl Math Comput. 2009;209(2):425-9.

[44] Wazwaz AM. Solitary wave solutions for modified forms of Degasperis-Procesi and Camassa-Holm equations. Phys Lett A. 2006;352(6):500-4.

[45] Wazwaz AM. New solitary wave solutions to the modified forms of Degasperis-Procesi and Camassa-Holm equations. Appl Math Comput. 2007;186(1):130-41.

[46] Yusufoğlu E. New solitonary solutions for modified forms of DP and CH equations using Exp-function method. Chaos Solitons Fractals. 2009;39(5):2442-7.

[47] Abro KA. Numerical study and chaotic oscillations for aerodynamic model of wind turbine via fractal and fractional differential operators. Numer Methods Partial Differential Eq. 2020:1-15.

[48] Abro KA, Qureshi S, Atangana A. Mathematical and numerical optimality of non-singular fractional approaches on free and forced linear oscillator. Nonlinear Eng. 2020;9(1):449-56.

[49] Takasar H, Aziz UA. Kashif Ali Abro, Muhammad Ozair, Mehwish Manzoor, A mathematical and parametric study of epidemiological smoking model: a deterministic stability and optimality for solutions. Eur Phys J Plus. 2021;136(1):11.

[50] Imran QM, Abro KA, Muhammad AS, Asif AS. Functional shape effects of nanoparticles on nanofluid suspended in ethylene glycol through Mittage-Leffler approach. Phys Scr. 2020;96(2):025005.

[51] Samuel O. Adesanya, Basma Souayeh, Mohammad RahimiGorji, M.N. Khan, O.G. Adeyemi, Heat irreversibiility analysis for a couple stress fluid flow in an inclined channel with isothermal boundaries. J Taiwan Inst Chem Eng. 2019;101:2518.

[52] Kashif AA, Jose FG. Fractional modeling of fin on non-Fourier heat conduction via modern fractional differential operators. Arab J Sci Eng. 2021;46(3):2901-10.

[53] Mohyud-Din ST, Hamid M, Usman M, Kanwal A, Zubair T, Wang W, et al. Rotating flow of nanofluid due to exponentially stretching surface: an optimal study. J Algorithm Comput Technol. 2019;13:1-14.

[54] Syed TS, Abro KA, Sikandar A. Role of single slip assumption on the viscoelastic liquid subject to non-integer differentiable operators. Math Methods Appl Sci. 2021;44(7):1-16.
[55] A Atangana, Araz S I, New concept in calculus: Piecewise differential and integral operators, Chaos, Solitons \& Fractals 2021;145:110638. https://doi.org/10.1016/j.chaos.2020.110638.

[56] Abdon A, Seda IA. Extension of Atangana-Seda numerical method to partial differential equations with integer and noninteger order. Alex Eng J. 2020 Aug;59(4):2355-70.

[57] Kashif AA, Abdon A. Jose Francisco Gomez-Aguilar, Role of bi-order Atangana-Aguilar fractional differentiation on Drude model: an analytic study for distinct sources. Opt Quantum Electron. 2021;53(4):177.

[58] Hamid M, Usman M, Khan ZH, Wang W, Ahmad R. Dual solutions and stability analysis of flow and heat transfer of Casson fluid on a stretching sheet with convective boundary condition. Phys Lett A. 2019;383:2400-8.

[59] Imran S, Nehad AS, Kashif AA. Thermography of ferromagnetic Walter's-B fluid through varying thermal stratification. $S$ Afr J Chem Eng. 2021;36:118-26.

[60] Riaz QAS, Awan AU, Abro KA, A mathematical model for thermography on viscous fluid based on damped thermal flux. $Z$ Naturforsch A. 2021;76(3):285-94.

[61] Aatangana A. Extension of rate of change concept: from local to nonlocal operators with applications. Results Phys. 2020;19:103515.

[62] Abdon A, Goufo EFD. Cauchy Problems With FractalFractional Operators And Applications To Ground Water Dynamics. Fractals. 2020;28(8);2040043. https://doi.org/10.1142/S0218348X20400435.

[63] Aziz UA, Attia S, Abro KA, Muhammad O, Takasar H. Dynamical Aspects of Smoking Model with Cravings to Smoke. Nonlinear Eng. 2021;10(1):91-108.

[64] Hamid M, Usman M, Khan ZH, Haq RU, Wang W. Heat transfer and flow analysis of Casson fluid in a partially heated trapezoidal cavity. Int Commun Heat Mass Transf. 2019;108:104284.

[65] Aziz UA, Mashal A, Naeem U, Sohail N, Kashif AA. Thermal analysis of oblique stagnation point low with slippage on second-order flluid. J Therm Anal Calorim. 2021. https://doi.org/10.1007/s10973-021-10760-z.

[66] Khader M, Saad K, Hammouch Z, Baleanu D. A spectral collocation method for solving fractional KdV and KdV-Burgers equations with non-singular kernel derivatives. Appl Numer Math. 2020;161:137-46.

[67] Arfan M, Shah K, Abdeljawad T, Hammouch Z. An efficient tool for solving two-dimensional fuzzy fractionalordered heat equation. Numer Methods Partial Differ Equ. 2020; https://doi.org/10.1002/num.22587.

[68] Muhammad T, Aziz UA, Kashif AA. Extraction of optical solitons in birefringent fibers for Biswas-Arshed equation via extended trial equation method. Nonlinear Eng. 2021;10(1):14658.

[69] Kashif AA, Abdon A. A computational technique for thermal analysis in coaxial cylinder of one-dimensional flow of fractional Oldroyd-B nanofluid, Int J Ambient Ener. 2021. https://doi.org/10.1080/01430750.2021.1939157.

[70] Atangana A, Seda IA. New numerical approximation for Chua attractor with fractional and fractal-fractional operators. Alex Eng J. 2020;59(5):3275-96.

[71] Kashif AA, Abdon A, Ali RK. Dynamical behavior of fractionalized simply supported beam: an application of fractional operators to Bernoulli-Euler theory. Nonlinear Eng. 
2021. https://doi.org/10.1515/nleng-2021-0017.

[72] Hamid M, Khan ZH, Khan WA, Haq RU. Natural convection of water-based carbon nanotubes in partially heated rectangular fin-shaped cavity with inner cylindrical obstacle. Phys Fluids. 2019;31(10):103607.

[73] Kashif AA, Abdon A. Strange Attractors and Optimal Analysis of Chaotic Systems based on FractalFractional Differential Operators, Int J Model Simul. 2021. https://doi.org/10.1080/02286203.2021.1966729.

[74] Saad KM, Gomez-Aguilar JF, Almadiy AA. A Fractional Numerical Study on a Chronic Hepatitis C Virus Infection Model with Immune Response. Chaos Solit Fractals. 2020;139:110062. 